\title{
Short-term prophylaxis with ciprofloxacin in extended 16- core prostate biopsy
}

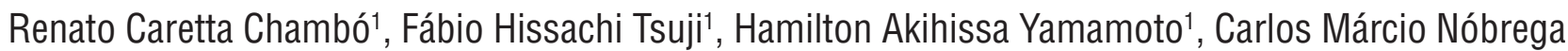 \\ de Jesus ${ }^{1}$
}

${ }^{1}$ Botucatu Medical School, São Paulo State University (UNESP), Botucatu, São Paulo, Brazil

\section{ABSTRACT}

Objective: To evaluate the safety, efficacy and possible complications of 16-core transrectal prostate biopsies using two doses of ciprofloxacin for prophylaxis of infectious complications.

Materials and Methods: Sixteen-core prostate biopsies were performed on a number of patients with different signs of potential prostate cancer. Complications were assessed both during the procedure and one week later. After the procedure, urine samples were collected for culture. The rate of post-biopsy complications, hospital visits and hospitalizations were also analyzed. Ciprofloxacin (500 mg) was administered two hours before, and eight hours after the procedure.

Results: The overall rate of post-biopsy complications was $87.32 \%$, being $5.4 \%$ of those considered major complications due to hemorrhage, or to urinary retention. Eight patients required hospital treatment post-biopsy. Fever occurred in just one patient (0.29\%). There was no incidence of orchitis, epididymitis, prostatitis, septicemia, hospitalization, or death. The urine culture showed positive results in five patients (2.15\%). Conclusion: One-day prophylaxis with ciprofloxacin proved to be safe and effective in the prevention of infectious complications following 16-core prostate biopsies.

\section{ARTICLE INFO}

\section{Key words:}

prostate; prostatic neoplasms; biopsy; complications

Int Braz J Urol. 2015; 41: 46-56

Submitted for publication: February 09, 2014

Accepted after revision:

May 13, 2014

\section{INTRODUCTION}

Excluding skin cancers, prostate cancer ( $\mathrm{PCa})$ is the most common cancer in men and the second cause of death, only after lung cancer. The estimated new cases of PCa and death in the United States (USA) in 2014 were 233,000 and 29,480, respectively (1). In Brazil, the number of deaths in 2011 was 13,129 and the estimated new cases for the year 2014 will be 68,800 (2).

The method of choice for final diagnosis of Pca is transrectal ultrasound (TRUS)-guided biopsy (2). Despite various studies in the literature that demonstrate low rates of complications and good tolerance to the procedure $(3,4)$, it is still considered invasive and not entirely free of complications. For some patients, it is an arduous and painful exam. Furthermore, the procedure cannot guarantee the absence of Pca, even with a negative result. Due to the aforementioned issues, there is great interest in making the exam as safe, fast and efficient as possible, along with the lowest rates of complications achievable.

The main complications related to prostate biopsy may be immediate, such as rectal bleeding (1.3 to $33.1 \%$ ), hematuria (13 to $65 \%$ ), vaso-vagal response (0 to 2.8\%), and delayed, such as fever (1.7 to $6.6 \%$ ), hemospermia (5.1 to 50.4\%), persistent 
dysuria (0 to $7.2 \%$ ), infection (2.5 to $9.2 \%$ ), acute prostatitis (0 to $1.8 \%$ ) and urosepsis (0 to $8 \%$ ) $(5,6)$.

There are many measures that can be taken in prostate biopsies in order to minimize post-procedural complications. Such measures include prior evaluation of patient co-morbidities; intestinal preparation; administration of prophylactic antibiotics; indication of the ideal number and location of biopsies to be performed; use of anesthesia or sedation; and appropriate room with all the material necessary for the intervention on emergency situations $(6,7)$.

Antibiotic prophylaxis generally reduces the risk of infectious complications following TRUS guided biopsy (8). Oral quinolones are the most common prophylactic antibiotics, either alone or in combination with other antibiotics; optimal dosing and treatment period vary, but in the last few years increased resistance to quinolones has been reported associated with a rise in severe infectious complications after biopsy $(4,9)$.

The aim of this present study was to evaluate the complications, possible risk factors, outcomes, safety and efficacy of TRUS guided biopsy with removal of 16 fragments, using two doses of ciprofloxacin as prophylaxis against infectious complications.

\section{MATERIALS AND METHODS}

The present prospective study was conducted from January 2011 to February 2012 within the Department of Urology, Botucatu Medical School - UNESP after the approval of the Research Ethics Committee. A sample of 351 patients was submitted consecutively to TRUS prostate biopsies. The criteria for inclusion in the study were: digital rectal exams suggestive of neoplasia; elevated prostate specific antigen (PSA) (higher than $4.0 \mathrm{ng} / \mathrm{mL}$ in men over the age of 55 and higher than $2.5 \mathrm{ng} / \mathrm{mL}$ in men under the age of 55); PSA density greater than 0.15 $\mathrm{ng} / \mathrm{mL}$; and annual increase rate of PSA higher than $0.75 \mathrm{ng} / \mathrm{mL}$. Carriers of coagulopathies, individuals with urinary tract infections (whether diagnosed at the time of biopsy or in treatment), and those who refused informed written consent were excluded from the study.
After consulting the patient's medical records, parameters such as age, race, serum total PSA (current and previous), free PSA, free PSA / total PSA and biopsy indication were analyzed. Prior to ultrasound, a digital rectal exam was conducted. Prostate volume and nodule presence were determined via ultrasound. Complications during the procedure were rectal bleeding, urethral bleeding, hypotension, vaso-vagal response, hypoxia, nausea, vomiting, pain and urinary retention.

The biopsy was performed on an outpatient basis, in a room equipped with all material necessary for emergency intervention. Sedation and anesthesia were realized by the administration of $50 \mathrm{mcg}$ fentanyl citrate and $5 \mathrm{mg} \mathrm{mi-}$ dazolam. The biopsies were performed by two experienced urologists. On the morning of the procedure, rectal enema was performed with 250 $\mathrm{mL}$, and antibiotic prophylaxis was achieved with the oral administration of ciprofloxacin 500 mg two hours prior to the procedure, and again eight hours after it. The procedure was performed with the patient in the left lateral position with his thighs flexed. The procedure was performed using a Dornier transrectal ultrasound equipment, with a $6.5 \mathrm{MHz}$ multiplanar probe, auto-fire gun and 18 gauge needle.

Shortly after the biopsy, urine samples were collected for culture.

Sixteen punctures were performed, obtaining cores bilaterally from the following regions of the prostate: the base, middle third, apex, medial (transitional zone), and latero-lateral. After collecting these cores, six additional punctures were made bilaterally in the more lateral regions of the base, the middle third, and the apex (Figure-1).

The positivity for diagnosis of prostate cancer was assessed as well as the overall rate of post-biopsy complications.

Seven days after the procedure, the patients returned to the clinics where they completed a questionnaire to assess possible complications resulting from the procedure such as pain, fever, hematuria, dysuria, hemospermia, and rectal bleeding. Visits to the hospital as well as hospitalizations were also analyzed. 
Figure 1 - Regions where punctures were made to collect prostate cores: 1. right base, 2. right middle third, 3. right apex, 4. latero-lateral right, 5. right medial, 6. left base, 7. left middle third, 8. Left apex, 9. latero-lateral left, 10. left medial, 11. right base, 12. right middle third, 13. right apex, 14. left base, 15. left middle third and 16 . left apex.

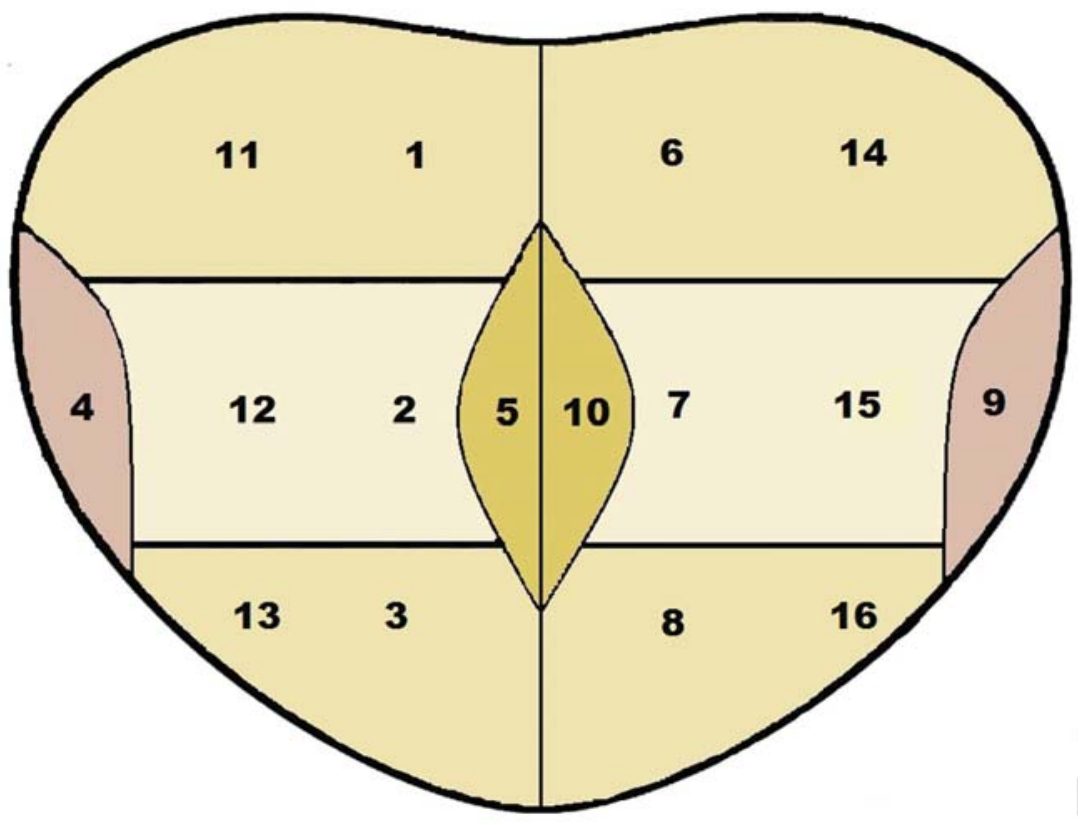

\section{RESULTS}

Of the 351 patients who underwent the procedure, the immediate complications (during and after biopsy) were rectal bleeding, intense pain, urinary retention, urethral bleeding, hypotension, vaso-vagal response (sweating, hypotension, bradycardia), nausea and vomiting (Table-1).

In 347 patients, long-term complications were hematuria, hemospermia, rectal bleeding, dysuria, pain and fever. There were no cases of sepsis and death (Table-2). We had a loss of four patients who did not return to the clinic to complete the questionnaire of long-term complications.

Positive results for $\mathrm{PCa}$ with the collection of 16 cores were $30.48 \%$.

The overall rate of post-biopsy complications was $87.32 \%$, with $5.4 \%$ of those being considered major complications, and the rest minor complications.

Eight patients sought emergency hospital care after the biopsy due to urinary retention, intense hematuria and urethral bleeding (Table-3).
After the prostate biopsy procedure, urine samples were collected for culture in 232 patients, five of which were positive (2.15\%) (Table-4). Of the 351 patients submitted to the prostate biopsy, eight patients $(2.27 \%)$ were catheterized with an indwelling catheter, and did not have urine samples collected.

\section{DISCUSSION}

Prostate cancer is a neoplasm with particularly insidious onset, and as with any other malignant neoplasm, there is great concern to stablish an early diagnosis. With the introduction of PSA testing in the screening for prostate cancer, there was an important advancement in the early diagnosis of the disease, making possible the detection of subclinical neoplasms in many more patients.

TRUS guided biopsy is the most accepted method for the diagnosis of prostate cancer (10). Although it is the ideal method for obtaining prostatic cores for analysis, TRUS biopsy of the prostate is considered an invasive procedure that 
Table 1 - Immediate complications (during and after biopsy).

\begin{tabular}{lcc}
\hline Immediate complications & N (351) & $\%$ \\
\hline Rectal bleeding & 30 & 8.54 \\
Intense pain & 17 & 4.84 \\
Urinary retention & 16 & 4.56 \\
Urethral bleeding & 9 & 2.56 \\
Hypotension & 3 & 0.85 \\
Vaso-vagal response (sweating, hypotension and bradycardia) & 2 & 0.57 \\
Nausea & 1 & 0.28 \\
Vomiting & 1 & 0.28 \\
Hypoxia & 1 & 0 \\
\hline
\end{tabular}

Table 2 - Delayed complications.

\begin{tabular}{lcccc}
\hline & $\mathrm{N}(347)$ & $\%$ & Average (days) & SD \\
\hline Hematuria & 226 & 65.13 & 2.50 & 3.38 \\
Hemospermia & 155 & 44.67 & 4.68 & 7.74 \\
Rectal bleeding & 78 & 22.48 & 0.61 & 1.86 \\
Dysuria & 51 & 14.70 & 0.65 & 2.09 \\
Pain & 33 & 9.54 & 0.27 & 1.17 \\
Fever & 1 & 0.29 & 0.01 & 0.12 \\
Sepsis & 0 & 0 & 0 & 0 \\
Death & 0 & 0 & 0 & 0 \\
\hline
\end{tabular}

Table 3 - Hospital visits post-biopsy.

\begin{tabular}{lcc}
\hline Hospital visits post-biopsy & $\mathrm{N}(347)$ & $\%$ \\
\hline Urinary retention & 5 & 1.44 \\
Intense hematuria & 2 & 0.57 \\
Urethral bleeding & 1 & 0.28 \\
\hline
\end{tabular}

Table 4 - Urine culture post-biopsy.

\begin{tabular}{lcc}
\hline Urine culture post-biopsy & $\mathrm{N}(232)$ & $\%$ \\
\hline Mixed flora & 2 & 0.86 \\
E. coli & 1 & 0.43 \\
Citrobacter freundii & 1 & 0.43 \\
Morganella morganii & 1 & 0.43 \\
\hline
\end{tabular}

is uncomfortable for the patient $(6,11)$. Considering the fact that the vast majority of men subjected to the exam show no signs of cancer, there is great concern that the procedure be as safe and effective as possible. Thus, complications should be minimized whenever possible. These complications also translate into costs. In USA, it is estimated that about $1,000,000$ prostate biopsies are carried out per year (4). If one were to consider, hypothetically, if just $1 \%$ of those patients were to experience complications that required medical care and the interruption of their professional lives, the inherent cost of the procedure - both societally and on the individual level - are considerably alarming.

The positivity for $\mathrm{CaP}$ with collecting 16 fragments was $30.48 \%$; in the literature the rate is 30 to $40 \%$ (12). 
The overall rate of complications after biopsy was found to be $87.32 \%$ in our study. $5.4 \%$ of those complications were considered to be major, such as hematuria and rectal bleeding for more than 7 days, infectious complications, urinary retention and death. Minor complications are mostly self-limited, resolving days after the biopsy, not causing increased morbidity to the patient. These data were also reported by other authors, such as Rodriguez and Terris (11) and Jesus et al. (6). This is important because we have given a higher probability of having some kind of complication, and patients have the right and our obligation to be alerted to this fact.

Despite occurring with great frequency, the minor complications associated with this procedure are little discussed in the literature, with the infectious complications being given much greater focus (6). Amongst the most common minor complications are those of hemorrhagic origin, such as hematuria. The results of the present study demonstrate an incidence of $65.13 \%$ of patients with hematuria, values consistent with those found in the literature $(6,13,14)$.

The hemospermia in this study was the second cause of hemorrhagic complications corresponding to $44.67 \%$ of patients undergoing biopsy, also compatible with the data values from the literature $(6,14)$. The reported rate of hemospermia varies widely among studies, this variation may reflect cultural issues, absence of sexual activity, social stigma, or different perceptions of importance as well as differences in data collection among studies (timing and method of assessment) (9). Hemospermia is the type of hemorrhagic complications that worries and frightens the patient submitted to prostate biopsy and more common in sexually active patients.

As the third most common complication in the present study, rectal bleeding was reported in $22.48 \%$ of the patients submitted to biopsy. Other studies relate an incidence of rectal bleeding that varies from $1.3 \%$ to $37.1 \%$ of biopsied patients (6, $11,13,14)$. Almost all patients had rectal bleeding after prostate biopsy, assessment of immediate bleeding was subjective. Patients who had little bleeding were not considered in the study and patients with severe rectal bleeding who underwent treatment or observation were considered.
Infectious complications are less common than hemorrhagic, but present a greater risk of morbidity to the patient. Several recent studies describe increased rates of hospitalization after prostate biopsy, specifically because of infectious complications. Severe sepsis has been reported in $0.1 \%$ to $3.5 \%$ of patients, with Escherichia coli being the most common bacteria involved $(4,13$, 15). In the present study, only one patient $(0.29 \%)$ presented with fever and chills on the second day post-biopsy, which lasted only one day after beginning a 7-day course of ciprofloxacin. 51 patients (14.70\%) presented with dysuria, but without the presence of infection, and no patient developed sepsis. Some authors consider asymptomatic bacteremia an infectious complication, though the majority only considers bacterial presence a complication when accompanied by clinical symptoms (16). Dysuria and pollakiuria are symptoms that are only considered infectious when accompanied by other symptoms of infection (positive urine culture, fever, chills, orchitis, or prostatis). Dysuria and pollakiuria can be the result of irritative factors after prostate biopsies such as edema or clots that obstruct the passage of urine through the urethral canal.

The post-biopsy urine culture was positive in five patients $(2.15 \%)$ from a total of 232 samples. Only one patient with positive urine culture evolved with symptomatology, developing urinary retention. Although not frequently seen, asymptomatic bacteriuria was reported by Fong et al. (17) in $7 \%$ of the patients in their study in which two types of antibiotics were compared. The presence of positive urine culture is associated with the use of antibiotic prophylaxis, the type of antibiotic used, and the presence of urinary tract infection prior to biopsy $(8,17)$.

One strategy to reduce infectious complications is rectal cleansing; a Cochrane review concluded that enema plus antibiotics reduced the risk of bacteremia (relative risk [RR]: 0.25; 95\%CI, 0.08-0.75) compared with antibiotics alone (7). The problem of infection after TRUS-guided biopsy has long been recognized in many studies, with bacteraemia occurring in almost all and bacteriuria in $13-36 \%$ of men when a placebo or no antibiotic prophylaxis is used (7-9). 
Currently, the use prophylactic antibiotics to minimize infective complications are routinely used, but such therapy does not completely eliminate infection and there is no consensus about antibiotic choice, dose, route of administration and duration of therapy (9). In a systematic review, Zani et al. (7) showed that antibiotic prophylaxis is effective in the prevention of infectious complications after prostate biopsy and that a variety of classes of antibiotics are effective. The quinolone class (ciprofloxacin) was the most widely researched class, with the greatest number of studies and subjects dedicated to its research. There were no definitive studies to confirm that long duration antibiotic courses (three days) are superior to short duration courses (one day), or that multiple dose treatment is superior to single dose treatment.

Antibiotic prophylaxis most commonly uses fluoroquinolones (ciprofloxacin), largely due to a broad spectrum of antibacterial activity, including most aerobic microorganisms residing in the bowel, good oral bioavailability (70\% to $80 \%$ ), extended half-life (4 to 5 hours), high concentration in both urine and prostate tissue, and post-antibiotic effect suppressing bacterial growth lasting for 2 to 6 hours $(8,18)$. Thus, ciprofloxacin becomes a logical candidate for the prophylaxis of urinary tract infections in patients undergoing prostate biopsy. However, recent studies have described an increase in infections after prostate biopsy by fluoroquinolone-resistant E. coli $(19,20)$.

A new strain of E. coli recently emerged from phylogenetic group B2, sequence type 131 (ST131), characterized by its ability to combine a set of extra-bowel virulence factors with antimicrobial resistance, principally against fluoroquinolone $(21,22)$. Recent studies have emphasized that E. Coli ST131 is capable of spreading in the community and rates of fluoroquinolone-resistant bowel colonization can rise amongst men who undergo prostate biopsies $(23,24)$.

An increase in fluoroquinolone-resistant E. coli strains has been observed in certain localities, remaining below 5\% in most places, however, certain areas have seen more significant increases, such as São Paulo, Brazil, wherein the density of these strains has continued to grow even further in recent years (19).
Quinolone-resistant strains of E. coli will become even more common in the future. For areas in which the rate of infection from prostate biopsy remains high, the exclusive use of alternative prophylactic antibiotic regiments, or alternatives used in combination with fluoroquinolones, may be useful in the reduction of rates of complications $(25,26)$.

The low rate of infectious complications observed in our study may be due to a population less exposed to antibiotics and a lower incidence of $\mathrm{E}$. coli strains resistant to ciprofloxacin, demonstrating the efficacy and safety of short-term prophylaxis with ciprofloxacin in our location.

Eight patients $(2.30 \%)$ in a total of 347 patients were hospitalized, five of them due to urinary retention. In our study there was no hospitalization resulting from post- biopsy complications. In literature hospitalization rates range from 0.1 to $3.4 \%$ (5, $6,11,13,15)$.

In our study, 16 patients (4.56\%) presented with urinary retention, a number significantly greater than those seen in comparable studies. Only in the study of Deliveliotis et al. (27) occurred a similar rate (4.6\%) of urinary retention. The cause of urinary retention can be explained perhaps by collecting fragments in transition zones near the prostatic urethra, prostate volume, lower urinary tract symptoms (LUTS) pre biopsy or the number of cores collected. In our study, we collected two cores from the transition zone (one core bilaterally), which may have resulted in injury and inflammation near the urethra, thus explaining the increase in rates of urinary retention. Biopsy in the transition zone has a low rate of detection of PCa (1.8\%), and does not improve the number of positive re-biopsies. There is currently no indication for the collection of cores from the transition zone (28). In our sample of 351 patients, only one patient $(0.28 \%)$ was diagnosed with PCa due to collection from the transition zone, reinforcing the lack of necessity for the biopsy of this region. Raaijmakers et al. (13) demonstrated that an increase in prostate volume is associated with hematuria of over three days' duration $(\mathrm{p}<0.001)$ and acute urinary retention $(p=0.009)$. The mean prostate volume in the 16 patients who developed urinary retention in our study was $50.12 \mathrm{~cm}^{3}$, which is not statistically different from patients who did not experience this complication. According to the data obtained in this 
study, Rodriguez and Terris (11) did not demonstrate any association with prostate volume and morbidity. Zisman et al. (29) reported a relationship between prostate biopsy and LUTS, a transient voiding impairment or acute urinary retention might be precipitated by biopsy, especially in patients with a transition zone volume $>42 \mathrm{~mL}$ and with a baseline IPSS of $>20$. In our study we did not performed the evaluation of LUTS in pre biopsy period. It seems logical that the greater the number of cores collected, the greater the damage to the prostate, but in many studies comparing biopsies with varying numbers of cores collected, there were no statistically significant differences regarding complications associated with prostate biopsy $(5,30,31)$.

\section{CONCLUSIONS}

Prophylaxis with a single-day course of ciprofloxacin in this study proved to be effective, with low rates of infectious complications, even in extended biopsies. These findings demonstrate that ciprofloxacin remains the gold-standard drug for antibiotic prophylaxis in this procedure.

\section{CONFLICT OF INTEREST}

None declared.

\section{REFERENCES}

1. Siegel R, Ma J, Zou Z, Jemal A. Cancer statistics, 2014. CA Cancer J Clin. 2014:64:9-29. Erratum in: CA Cancer J Clin. 2014;64:364.

2. Instituto Nacional do Câncer [Internet]. Rio de Janeiro: INCA. 2014. Estimativa 2014 - Incidência de câncer no Brasil. [cited 2011 Jun 01]. Available from: http://www2.inca.gov. br/wps/wcm/connect/tiposdecancer/site/home/prostata

3. Kakehi Y, Naito S; Japanese Urological Association. Complication rates of ultrasound-guided prostate biopsy: a nation-wide survey in Japan. Int J Urol. 2008;15:319-21.

4. Loeb S, Carter HB, Berndt SI, Ricker W, Schaeffer EM. Complications after prostate biopsy: data from SEERMedicare. J Urol. 2011;186:1830-4.

5. Djavan B, Waldert M, Zlotta A, Dobronski P, Seitz C, Remzi M, et al. Safety and morbidity of first and repeat transrectal ultrasound guided prostate needle biopsies: results of a prospective European prostate cancer detection study. J Urol. 2001;166:856-60.
6. de Jesus CM, Corrêa LA, Padovani CR. Complications and risk factors in transrectal ultrasound-guided prostate biopsies. Sao Paulo Med J. 2006;124:198-202.

7. Zani EL, Clark OA, Rodrigues Netto N Jr. Antibiotic prophylaxis for transrectal prostate biopsy. Cochrane Database Syst Rev. 2011;(5):CD006576.

8. Kapoor DA, Klimberg IW, Malek GH, Wegenke JD, Cox CE, Patterson AL, et al. Single-dose oral ciprofloxacin versus placebo for prophylaxis during transrectal prostate biopsy. Urology. 1998;52:552-8.

9. Heidenreich, P.J. Bastian, J. Bellmunt, M. Bolla, S. Joniau, M.D. Mason, et al. Guidelines on Prostate Cancer, European Association of Urology, 2012. Available at. http://www.uroweb.org/gls/pdf/08\%20Prostate $\% 20$ Cancer_LR\%20March\%2013th\%202012.pdf

10. Dall'Oglio MF, Crippa A, Faria EF, Cavalhal GF, et al. Diretrizes de Cancer de Próstata. SociedadeBrasileira de Urologia. 2011. Available at: http://sbues.org.br/ diretrizes/cancer_prostata.pdf

11. Rodríguez LV, Terris MK. Risks and complications of transrectal ultrasound guided prostate needle biopsy: a prospective study and review of the literature. J Urol. 1998;160:2115-20.

12. Escudero Bregante JF, López Cubillana P, Cao Avellaneda E, López López Al, Maluff Torres A, López González PA, et al. Clinical efficacy of prostatic biopsy. Experience in our center from 1990 to 2002. Actas Urol Esp. 2008;32:713-6.

13. Raaijmakers R, Kirkels WJ, Roobol MJ, Wildhagen MF, Schrder FH. Complication rates and risk factors of 5802 transrectal ultrasound-guided sextant biopsies of the prostate within a population-based screening program. Urology. 2002;60:826-30.

14. Utrera NM, Sánchez AT, Rodríguez-Antolín A, MartínParada A, Lora D, Passas J, et al. Saturation biopsies for prostate cancer detection: effectiveness, safety and predictive factors. Arch Esp Urol. 2011;64:421-6.

15. Nam RK, Saskin R, Lee Y, Liu Y, Law C, Klotz LH, et al. Increasing hospital admission rates for urological complications after transrectal ultrasound guided prostate biopsy. J Urol. 2013;189(1 Suppl):S12-7; discussion S17-8.

16. Roach MB, Figueroa TE, McBride D, George WJ, Neal DE Jr. Ciprofloxacin versus gentamicin in prophylaxis against bacteremia in transrectal prostate needle biopsy. Urology. 1991;38:84-7.

17. Fong IW, Struthers N, Honey RJ, Simbul M, Boisseau DA. A randomized comparative study of the prophylactic use of trimethoprim-sulfamethoxazole versus netilmycinmetronidazole in transrectal prostatic biopsy. J Urol. 1991;146:794-7.

18. Tolkoff-Rubin NE, Rubin RH. Ciprofloxacin in management of urinary tract infection. Urology. 1988;31:359-67. 
19. Kiffer CR, Camargo EC, Shimakura SE, Ribeiro PJ Jr, Bailey TC, Pignatari AC, et al. A spatial approach for the epidemiology of antibiotic use and resistance in communitybased studies: the emergence of urban clusters of Escherichia coli quinolone resistance in Sao Paulo, Brasil. Int J Health Geogr. 2011;10: 17.

20. Zaytoun OM, Vargo EH, Rajan R, Berglund R, Gordon S, Jones JS. Emergence of fluoroquinolone-resistant Escherichia coli as cause of postprostate biopsy infection: implications for prophylaxis and treatment. Urology. 2011;77:1035-41.

21. Johnson JR, Johnston B, Clabots C, Kuskowski MA, Castanheira M. Escherichia coli sequence type ST131 as the major cause of serious multidrug-resistant E. coli infections in the United States. Clin Infect Dis. 2010;51:286-94.

22. Rogers BA, Sidjabat HE, Paterson DL. Escherichia coli 025bST131: a pandemic, multiresistant, community-associated strain. J Antimicrob Chemother. 2011;66:1-14.

23. Peirano G, Costello M, Pitout JD. Molecular characteristics of extended-spectrum beta-lactamase-producing Escherichia coli from the Chicago area: high prevalence of ST131 producing CTX-M-15 in community hospitals. Int J Antimicrob Agents. 2010;36:19-23.

24. Williamson DA, Roberts SA, Paterson DL, Sidjabat H, Silvey A, Masters J, et al. Escherichia coli bloodstream infection after transrectal ultrasound-guided prostate biopsy: implications of fluoroquinolone-resistant sequence type 131 as a major causative pathogen. Clin Infect Dis. 2012;54:1406-12.

25. Sieber PR, Rommel FM, Theodoran CG, Hong RD, Del Terzo MA. Contemporary prostate biopsy complication rates in community-based urology practice. Urology. 2007;70:498-500.

26. Chan ES, Lo KL, Ng CF, Hou SM, Yip SK. Randomized controlled trial of antibiotic prophylaxis regimens for transrectal ultrasound-guided prostate biopsy. Chin Med J (Engl). 2012;125:2432-5.
27. Deliveliotis C, John V, Louras G, Andreas S, Alargof E, Sofras F, et al. Multiple transrectal ultrasound guided prostatic biopsies: morbidity and tolerance. Int Urol Nephrol. 1999;31:681-6.

28. Pelzer AE, Bektic J, Berger AP, Halpern EJ, Koppelstätter F, Klauser A, et al. Are transition zone biopsies still necessary to improve prostate cancer detection? Results from the tyrol screening project. Eur Urol. 2005;48:916-21; discussion 921.

29. Zisman A, Leibovici D, Kleinmann J, Cooper A, Siegel Y, Lindner A. The impact of prostate biopsy on patient wellbeing: a prospective study of voiding impairment. J Urol. 2001;166:2242-6.

30. Nomikos M, Karyotis I, Phillipou P, Constadinides C, Delakas D. The implication of initial 24-core transrectal prostate biopsy protocol on the detection of significant prostate cancer and high grade prostatic intraepithelial neoplasia. Int Braz J Urol. 2011;37:87-93; discussion 93.

31. Irani J, Blanchet P, Salomon L, Coloby P, Hubert J, Malavaud $B$, et al. Is an extended 20-core prostate biopsy protocol more efficient than the standard 12-core? A randomized multicenter trial. J Urol. 2013;190:77-83.

Correspondence address:

Renato Caretta Chambó, MD

Botucatu Medical School, São Paulo State University

(UNESP)

Botucatu, São Paulo, Brazil

Rua Sete de Setembro, 860, Bairro Alto Cafeza

Marília, SP, 17502-020, Brazil

Telephone: + 5511 3433-1511

E-mail: renato.chambo@gmail.com 


\section{EDITORIAL COMMENT}

The referred manuscript is a large sample study and demonstrated good results in terms of low overall incidence of symptomatic urinary tract infection. These results denoted that ciprofloxacin still was effective in promoting antibiotic prophylaxis in the population evaluated despite the current increase in bacterial resistance rates faced by fluoroquinolones. This observation permits emphasize that population variability may play an important role on the selecting process of antibiotics for prophylaxis purposes. Consequently, knowledge of the bacterial resistance profile from the local population is paramount for optimizing post-biopsy infectious complications results. However, this study results have limitations, a control group was not designed for comparisons and therefore its evidence level was reduced to grade III. Other aspect is that post-biopsy urine culture was positive in four asymptomatic patients and was not considered as an infection event. Asymptomatic bacteriuria can lead to oligosymptomatic urine/prostate colonization causing eventual urinary tract infection onset in the future.

Is the era of empiric fluoroquinolones for prostate biopsy prophylaxis over?

Prostate biopsy is the gold-standard method for diagnosing prostate cancer. The procedure is most commonly performed through a transrectal approach, which can expose the genital and urinary tract to Gram-negative enterobacteria infection, especially caused by E. coli (1).

A Cochrane review on prophylaxis for transrectal prostate biopsy revealed a significant reduction in bacteriuria, urinary tract infection, bacteremia, fever and hospitalization after prostate biopsy with antibiotics compared to placebo. Definitive evidences of superiority of long-term or multiple-dose compared to short-term or single-dose antibiotic prophylaxis protocols were not demonstrated (2). Several reports have not shown significant difference between single-dose/1-day and 3-day prophylactic regimens (3-5). In addition, the American Urological Association has recommended antibiotic prophylaxis maintained for less than 24 hours in transrectal prostate biopsies (6). In this scenario, a short-term protocol may offer advantages of cost savings with potentially fewer drug related resistance.

Other efforts for reducing post-biopsy infection rates have been investigated. The use of enemas in association with antibiotic prophylaxis was also evaluated by Cochrane review. A reduced risk of bacteremia was identified when this association was applied compared to antibiotics alone, although no differences were found in fever or infection endpoints (2).

Patient-specific and procedure-specific characteristics were also described as possible potential risk factors for higher post-biopsy infection rates: increased comorbidity scores, untreated asymptomatic bacteriuria, history of prostatitis, urinary tract infection, prostate size, indwelling urinary catheters, presence of bladder stones, inadequately treated diabetes mellitus, number of biopsy cores and number of repeat biopsy procedures (7).

The choice of the prophylactic antibiotic type has been empirical and guided by the expected bacterial spectrum at the operative site, antibiotic susceptibility, drug pharmacokinetic and pharmacodynamics properties (8). Fluoroquinolones have traditionally been used as the primary prophylactic agent for prostate biopsy due to excellent urinary and prostatic penetration providing optimal coverage against key pathogens (9).

Despite the fact transrectal prostate biopsy has been widely considered a safe procedure for a long period of time and associated with low infectious complications rates, contemporary prospective and retrospective reports have currently shown a surprisingly increase on post-biopsy infectious rates from 1\% to $4 \%$ over the past fifteen years $(10,11)$. Parallel to this trend, studies have also shown a dramatically increase in the prevalence of fluoroquinolone-resistant E. coli strains (12). These findings have progressively changed the optimal scenario found by fluoroquinolones to effectively promote antibiotic prophylaxis in transrectal prostate biopsies.

Another important issue is that the previous controlled randomized trials that first evaluated the empirical use of antibiotic prophylaxis before transrectal prostate biopsies were performed when levels of resistance to commonly used antibiotics were generally low (2). 
In this respect, recent reports demonstrated presence of fluoroquinolone-resistant bacteria in $50-90 \%$ of patients with post-biopsy symptomatic infections. Additionally, the presence of fluoroquinolone-resistant pathogens in the rectal flora preoperatively, has been considered the most important risk factor for post-biopsy infection. The risk of harboring fluoroquinolone-resistant bacteria in the faeces was evaluated and increased remarkably on those who have received fluoroquinolones within the past 6 months or after international travel to countries with high levels of antibiotic resistance (12-14).

In this regard, non-randomized trials applied rectal swab cultures for preoperative assessment of rectal flora susceptibility and performed a targeted antimicrobial therapy based on its susceptibility profile. The targeted antibiotic prophylaxis was associated with a notable decrease in the incidence of infectious complications as well as a decrease in the overall cost of care (15).

Currently, these new attempts for a more individualized and optimized antibiotic prophylaxis based on the susceptibility profile of the rectal flora of each patient reached a new milestone of a new era on the way for reducing post biopsies infection rates. However, additional larger randomized prospective studies are still needed to further evaluate the efficacy and cost-effectiveness of this new strategy and compare it to the traditional empirical prophylaxis approach.

On the other hand, new biopsy technology as the MRI-transrectal ultrasonography (MRI-TRUS) fusion-guided-3D targeted biopsies has potential to reduce the number of repeated biopsies $(16,17)$. Consequently, it may reduce the amount of antibiotic used for prophylaxis and therefore possibly contribute for reducing antibiotic resistance in the future.

\section{REFERENCES}

1. Tobias-Machado M, Verotti MJ, Aragao AJ, Rodrigues A0, Borrelli M, Wroclawski ER. Prospective randomized controlled trial comparing three different ways of anesthesia in transrectal ultrasound-guided prostate biopsy. Int Braz J Urol. 2006;32:172-9; discussion 179-80.
2. Zani EL, Clark OA, Rodrigues Netto N Jr. Antibiotic prophylaxis for transrectal prostate biopsy. Cochrane Database Syst Rev. 2011;(5):CD006576.

3. Shigemura K, Tanaka K, Yasuda M, Ishihara S, Muratani T, Deguchi $\mathrm{T}$, et al. Efficacy of 1-day prophylaxis medication with fluoroquinolone for prostate biopsy. World J Urol. 2005;23:356-60.

4. Tobias-Machado M, Corrêa TD, De Barros EL, Wroclawski ER. Antibiotic prophylaxis in prostate biopsy. A comparative randomized clinical assay between ciprofloxacin, norfloxacin and chloramphenicol. Int Braz J Urol. 2003;29:313-9.

5. Aron M, Rajeev TP, Gupta NP. Antibiotic prophylaxis for transrectal needle biopsy of the prostate: a randomized controlled study. BJU Int. 2000;85:682-5.

6. American Urological Association Best Practice Policy Statement on Urologic Surgery Antimicrobial Prophylaxis 2008. (Reviewed and validity confirmed 2012, updated January 2014). Available at http://www.auanet.org/common/ pdf/education/clinical-guidance/Antimicrobial-Prophylaxis. pdf. Accessed May 5, 2014.

7. Loeb S, Vellekoop A, Ahmed HU, Catto J, Emberton M, Nam $\mathrm{R}$, et al. Systematic review of complications of prostate biopsy. Eur Urol. 2013;64:876-92.

8. Wagenlehner FM, Grabe M, Naber KG, Bjerklund Johansen TE, Naber CK, et al. Antibiotic prophylaxis in urology]. Urologe A. 2011;50:1469-78; quiz 1479-80.

9. Al-Hasan MN, Lahr BD, Eckel-Passow JE, Baddour LM. Antimicrobial resistance trends of Escherichia coli bloodstream isolates: a population-based study, 1998-2007. J Antimicrob Chemother. 2009;64:169-74.

10. Loeb S, Carter HB, Berndt SI, Ricker W, Schaeffer EM. Complications after prostate biopsy: data from SEERMedicare. J Urol. 2011;186:1830-4.

11. Loeb S, van den Heuvel S, Zhu X, Bangma CH, Schröder $\mathrm{FH}$, Roobol MJ. Infectious complications and hospital admissions after prostate biopsy in a European randomized trial. Eur Urol. 2012;61:1110-4.

12. Williamson DA, Roberts $S A$, Paterson DL, Sidjabat $H$, Silvey A, Masters J, et al. Escherichia coli bloodstream infection after transrectal ultrasound-guided prostate biopsy: implications of fluoroquinolone-resistant sequence type 131 as a major causative pathogen. Clin Infect Dis. 2012;54:1406-12.

13. Patel U, Dasgupta P, Amoroso P, Challacombe B, Pilcher $\mathrm{J}$, Kirby R. Infection after transrectal ultrasonographyguided prostate biopsy: increased relative risks after recent international travel or antibiotic use. BJU Int. 2012;109:1781-5.

14. Williamson DA, Masters J, Freeman J, Roberts S. Travelassociated extended-spectrum -lactamase-producing Escherichia coli bloodstream infection following transrectal ultrasound-guided prostate biopsy. BJU Int. 2012;109:E21-2. 
15. Taylor AK, Zembower TR, Nadler RB, Scheetz MH, Cashy JP, Bowen D, et al. Targeted antimicrobial prophylaxis using rectal swab cultures in men undergoing transrectal ultrasound guided prostate biopsy is associated with reduced incidence of postoperative infectious complications and cost of care. J Urol. 2012;187:1275-9.

16. Mouraviev V, Verma S, Kalyanaraman B, Zhai QJ, Gaitonde $\mathrm{K}$, Pugnale $\mathrm{M}$, et al. The feasibility of multiparametric magnetic resonance imaging for targeted biopsy using novel navigation systems to detect early stage prostate cancer: the preliminary experience. J Endourol. 2013;27:820-5.
17. Sonn GA, Natarajan S, Margolis DJ, MacAiran M, Lieu P, Huang J, et al. Targeted biopsy in the detection of prostate cancer using an office based magnetic resonance ultrasound fusion device. J Urol. 2013;189:86-91.

Dr. Marcos Tobias-Machado \& Dr. Igor Nunes-Silva Section of Urology, ABC Medical School São Paulo, SP, Brazil E-mail: tobias-machado@uol.com.br 